\title{
COMPREENDENDO O CORPO HUMANO POR MEIO DE METODOLOGIAS ATIVAS DE ENSINO-APRENDIZAGEM: ABORDAGEM EXTENSIONISTA COM ESCOLARES DO ENSINO MÉDIO
}

\section{UNDERSTANDING THE HUMAN BODY THROUGH ACTIVE TEACHING-LEARNING METHODOLOGIES: AN EXTENSION APPROACH WITH HIGH SCHOOLSTUDENTS}

Isaac de Andrade Santos (ORCID: 0000-0002-8816-6704) ${ }^{1}$

Ially Fraga Batista Andrade (ORCID: 0000-0002-0215-8253) ${ }^{1}$

Johnatan Weslley Araujo Cruz (ORCID: 0000-0002-5464-4114)

Gabriel Pereira de Oliveira Souza (ORCID: 0000-0003-3452-792X)

Marcela Ralin de Carvalho Deda Costa (ORCID: 0000-0003-1705-4833) ${ }^{2}$

Autor Correspondente Isaac de Andrade Santos

E-mail: isaacandrade97@gmail.com

Graduação em Fisioterapia, Universidade Federal de Sergipe.

${ }^{2}$ Departamento de Fisioterapia, Universidade Federal de Sergipe.

a) Financiamento: Pró-Reitoria de Extensão da Universidade Federal de Sergipe através da concessão de duas bolsas através do edital PIAEX $\mathrm{n}^{\circ}$ 9/2018 dos Programas Institucionais de Apoio à Extensão.

b) Trabalho oriundo das atividades desenvolvidas no projeto de extensão intitulado: "compreendendo o corpo humano: ensino da anatomia humana como forma de integração entre a escola e a universidade".

\section{RESUMO}

Objetivo: avaliar a eficácia das metodologias ativas de ensino-aprendizagem como ferramenta para o ensino da anatomofisiologia humana aos alunos do Ensino Médio (EM) de escolas públicas da cidade da Lagarto/SE. Métodos: trata-se de um estudo transversal com abordagem qualitativa e quantitativa, realizado entre julho e dezembro de 2018. As atividades de aprendizagem foram desenvolvidas semanalmente, por meio de módulos temáticos sobre Anatomofisiologia do Sistema Cardiorrespiratório (ASCR) e Reprodutor Humano (ASRH), com grupos de até 25 alunos. Para construção do aprendizado, foram utilizadas diversas metodologias ativas, com mensuração dessa aprendizagem mediante avaliações somativas e formativas iniciais e finais, além de avaliações das atividades propostas nas aulas. Resultados: participaram deste estudo 70 sujeitos, de modo que $89 \%$ eram do gênero feminino, e $50 \%$ cursavam a $3^{\text {a }}$ série do EM. Em relação aos módulos, todos os sujeitos participaram do módulo ASCR; destes, apenas 20 continuaram com as atividades do módulo ASRH. Após análises estatísticas por meio do Wilcoxon Test, foi evidenciado impacto positivo do uso de metodologias ativas no aprendizado por intermédio das notas obtidas na $2^{\mathrm{a}}$ avaliação somativa nos módulos ASCR $(\mathrm{p}<0.0001)$ e ASRH ( $\mathrm{p}=0.0038)$, assim como na avaliação formativa dos módulos ASCR ( $<<0.0001)$ e ASRH $(p=0.007)$. As avaliações das atividades propostas demonstram grande aceitabilidade dos alunos às novas formas de aprender. Conclusões: a utilização de metodologias ativas e seus diversos recursos para o ensino da anatomofisiologia demonstram grande eficácia e direcionam à construção de uma visão crítico- reflexiva por parte dos alunos, facilitando o processo ensino-aprendizagem.

Palavras-chave: Ensino Fundamental e Médio; Ensino; Anatomia; Fisiologia.

\begin{abstract}
Objective: evaluate the use of active teaching-learning methodologies as a tool for teaching human anatomophysiology to high school students (HS) from public schools in the city of Lagarto/SE. Methods: this is a cross-sectional study with a qualitative and quantitative approach, carried out between July and December 2018. The learning activities were developed weekly, through modules with the theme directed to the Anatomy and Physiology of the Cardiorespiratory System (APCRS) and Human Reproductive System (APHRS), with groups of up to 25 students. For the construction of learning, several active teaching-learning methodologies were used, with the measurement of this learning through initial and final summative and formative evaluations and placing of the activities proposed in classes. Results: 70 subjects participated in this study, so that $89 \%$ were female and $50 \%$ were in the 3 rd grade of HS. Regarding the modules, all subjects participated in the APCRS module and of these, only 20 continued with the activities of the APHRS module. After statistical analysis through the Wilcoxon Test, a positive impact of the use of active methodologies in learning was evidenced through the grades obtained in the 2nd summative assessment in the APCRS $(p<0.0001)$ and APHRS $(p=0.0038)$ modules, as well as in the formative assessment of APCRS $(p<0.0001)$ and APHRS $(p=0.007)$ modules. The evaluations of the proposed activities demonstrate great acceptability of students to new ways of learning. Conclusions: the use of active methodologies and their diverse resources for the teaching of human anatomy and physiology demonstrates great efficacy and directs the construction of a critical-reflexive view by the students, facilitating the teaching-learning process.
\end{abstract}

Keywords: Education, Primary and Secondary; Teaching; Anatomy; Physiology. 


\section{INTRODUÇÃO}

Em 384 a.C., Aristóteles descreveu o que seria a mais importante artéria do corpo, batizando-a como "aorta"1. Porém, antes mesmo disso, o ensino da anatomia já era amplamente conhecido e necessário nos meios acadêmicos e institucionais com intuito de fomentar a prática clínica e a pesquisa científica. Atualmente, o ensino da anatomia continua sendo uma ferramenta de grande importância nos meios supracitados, porém o foco mudou: agora a preocupação não é sobre a anatomia em si, mas, sim, como ensinar.

As aulas de anatomia sempre seguiram e ainda seguem o modelo bancário/tradicional de ensino-aprendizagem, o qual, segundo Paulo Freire ${ }^{2}$, diz respeito a um ensino no qual o professor é o retentor do conhecimento e que este tem o papel de transferi-lo aos seus alunos, não oferecendo a estes a autonomia necessária para a construção do seu aprendizado. Da mesma maneira, Behrens ${ }^{3}$ criticou o ensino no formato em que o estudante assume uma postura passiva e meramente reprodutora do conhecimento em vez de agente principal e ativo na construção do saber.

Em detrimento do insucesso dessas metodologias tradicionais de ensino e da necessidade de métodos que estimulem o ser crítico-reflexivo dos alunos, surgiram as metodologias ativas de ensino-aprendizagem baseadas na autonomia do estudante defendida por Paulo Freire ${ }^{4}$. A Aprendizagem Baseada em Problemas e a Problematização, demonstradas na prática pelo estudo de Aguiar et al. ${ }^{5}$, têm a finalidade de tornar a aprendizagem mais significativa e favorecer aos estudantes um formato de ensino autônomo e que lhes transformem em agentes sociais críticos-reflexivos.

Assim como o formato de ensinar é diferente, a maneira de avaliar os alunos também deve ser outra. Como é destacado no estudo de Aguiar et al. ${ }^{5}$, faz-se necessária a presença de avaliações contínuas e lineares, as quais ultrapassam o estudante, enquanto condição de avaliado, e alcançam os demais sujeitos e instrumentos pertencentes ao processo ensino-aprendizagem, como os professores/tutores e as atividades propostas.

Baseado nos conceitos acima descritos e considerando a necessidade do cenário educacional atual por metodologias e práticas de ensino que estimulem a autonomia dos estudantes nesse cenário de isolamento social, o presente estudo tem o objetivo de avaliar a eficácia do uso de metodologias ativas e inovadoras como ferramenta de ensino da anatomia humana para alunos do ensino médio de escolas públicas da cidade de Lagarto/SE.

\section{MÉTODOS}

Estudo transversal, com perspectiva quantitativa e qualitativa, realizado entre os meses de julho e dezembro de 2018 nas dependências físicas da Universidade Federal de Sergipe (UFS), campus universitário professor Antônio Garcia Filho, localizado na cidade de Lagarto/SE.

Com atendimento às normas da Resolução $n^{\circ}$ 466/12 do Conselho Nacional de Saúde e após aprovação do Comitê de Ética em Pesquisa da UFS com o parecer de $n^{\circ} 679.027$, foi entregue o Termo de Consentimento Livre e Esclarecido (TCLE) aos estudantes e aos respectivos responsáveis legais, quando menores de idade. Assim, participaram deste estudo os alunos da primeira, segunda e terceira série do ensino médio dos turnos matutino e vespertino, com idade entre 14 e 20 anos e matriculados nas instituições de ensino Colégio Estadual Silvio Romero e Colégio Estadual Abelardo Romero Dantas. 
Foram criadas quatro turmas para o desenvolvimento das atividades, as quais tinham capacidade máxima de 25 alunos e com a possibilidade de formação de duas turmas por instituição de ensino. As atividades foram organizadas, e os conteúdos programáticos foram divididos em dois módulos que aconteceram em sequência cronológica: fisiologia do sistema cardiorrespiratório e fisiologia dos aparelhos reprodutores humanos. As aulas aconteceram semanalmente, no turno oposto às atividades escolares obrigatórias dos estudantes. Os pesquisadores, graduandos da UFS, atuaram como tutores, orientando a execução das atividades e fomentando a participação dos sujeitos em sala de aula.

No módulo da fisiologia do sistema cardiorrespiratório, foram realizados quatro encontros com duração de duas horas. Foram discutidos: a anatomia do pulmão e coração; os princípios básicos da fisiologia respiratória e cardiovascular; e algumas disfunções, como o desenvolvimento e consequências do Infarto Agudo do Miocárdio (IAM) e a Doença Pulmonar Obstrutiva Crônica (DPOC).

As aulas de fisiologia dos sistemas reprodutores humano também foram realizadas em quatro encontros. Os temas versaram sobre anatomia do aparelho reprodutor masculino e feminino, introdução à fisiologia da reprodução, principais infecções sexualmente transmissíveis, métodos contraceptivos, diversidade de gênero e respeito à comunidade LGBTQIA+.

Durante a execução das aulas, alguns recursos, metodologias e estratégias foram utilizados com o objetivo de dinamizar e facilitar o processo ensino-aprendizagem, os quais incluem:

Chuva de ideias - Foi utilizada com a finalidade de evocar conhecimentos prévios dos alunos antes do início da aula. Os discentes eram orientados a escrever palavras ou frases no quadro branco que remetessem aos seus conhecimentos sobre os assuntos que iriam ser abordados. Assim, os tutores, no decorrer das aulas, realizavam associação entre o que foi exposto pelos alunos e o conteúdo programático.

Peças Anatômicas e Livros Atlas de Anatomia - Em todos os módulos, foram utilizadas peças sintéticas com demarcações das estruturas anatômicas que iriam ser trabalhadas durante as aulas para conhecimento e nomeação destas. Atlas e livros de anatomia foram usados para que, com a ajuda dos tutores, os alunos identificassem as estruturas correspondentes aos pontos demarcados nas peças. Além disso, as funções dessas estruturas anatômicas foram discutidas.

Recursos audiovisuais - Foram amplamente utilizados durante os dois módulos, especialmente ao final das discussões e atividades como maneira de dinamizar e consolidar o aprendizado. Os recursos comumente utilizados foram vídeos em animação 3D e imagens histológicas de microscopia.

Mapas conceituais - Mapas gigantes foram feitos com recortes de cartolinas contendo os nomes das estruturas e acontecimentos, nos quais os alunos tinham que montar esquemas sobre os assuntos, por exemplo: como funciona a circulação sistêmica e pulmonar, fluxo sanguíneo, sístole e diástole. Um tempo era estabelecido para execução da atividade, e, em seguida, os tutores realizavam a correção e a explicação do respectivo assunto. A elaboração de mapas conceituais no quadro branco pelos alunos no fim das aulas também era frequentemente solicitada, como forma de retomar os conteúdos discutidos.

Quiz - Durante a realização de algumas aulas, estes foram realizados com perguntas simples com respostas "sim ou não" sobre o conteúdo que estava sendo abordado, com o objetivo de revisar os conteúdos discutidos, assim como para avaliar a aprendizagem em construção.

Teste de Frequência Cardíaca - Foi uma dinâmica realizada no módulo sobre o aparelho cardiorrespiratório e teve como objetivo fixar e exemplificar o conteúdo sobre o Débito Cardíaco e a Frequência Cardíaca (FC). Os alunos foram divididos em dois grupos e, em seguida, foram escolhidos dois representantes, um de cada grupo, para um teste de verificação da FC, a qual foi aferida em repouso e após a corrida de uma certa distância, como forma de demonstrar a variação da $\mathrm{FC}$ em repouso e após esforço físico.

Pulmão reciclável - Foi construído 
durante o módulo sobre o aparelho cardiorrespiratório, com a utilização de cola quente, garrafa pet, tesoura, barbante, bexiga e canudos. Teve como objetivo facilitar o entendimento a respeito da mecânica respiratória e a função diafragmática.

Experimento Surfactante - Foi realizado um experimento no qual foi utilizado um bifásico de água e óleo para representar a função do líquido surfactante e a tensão superficial no alvéolo pulmonar.

Estruturas anatômicas de massa de modelar - Foi solicitada aos alunos a construção de estruturas anatômicas do aparelho reprodutor feminino a partir de massa de modelar, com base na própria criatividade e usando o atlas de anatomia para orientação. Posteriormente, os tutores solicitaram que os alunos explicassem sobre as estruturas construídas e as principais impressões sobre o conteúdo. Em casos necessários, os tutores realizaram correções ao longo das explicações dos estudantes.

Corrida dos espermatozoides Consiste em um jogo de tabuleiro (confecção própria) na forma simbólica de útero, trompas uterinas e ovários. No decorrer do jogo, o mesmo caminho que os espermatozoides percorrem até o momento da fecundação era seguido. Para a execução do jogo, cada turma foi dividida em dois grupos, e a progressão das casas era feita com base nos comandos e regras do jogo, bem como nos acertos das questões sobre o sistema reprodutor e fecundação.

Rodas de conversa - Foram utilizadas nos dois módulos com o objetivo de criar uma aproximação dos tutores com os alunos, bem como tirar dúvidas sobre os assuntos, abrir espaço para possíveis relatos de experiência, como também tornar as discussões mais leves.

Desenhos - Para trabalhar a temática da puberdade, foi solicitado que os alunos fizessem um desenho que descrevesse o que mais havia marcado o início da puberdade deles. Posteriormente, um bate papo em roda de conversa sobre o tema foi proposto e realizado.

Tellonym me - Consiste num aplicativo de perguntas anônimas que foi utilizado para tirar dúvidas sobre puberdade, sexualidade, doenças sexualmente transmissíveis e formas corretas de utilização dos contraceptivos.

Sociodrama - A peça teatral foi realizada pelos tutores no módulo dos aparelhos reprodutores humanos. Nela, existiram duas cenas, uma abordando sobre opiniões homofóbicas a respeito da comunidade LGBTQIA+ e outra a respeito das diferenças socioculturais associadas com o contexto da primeira cena. Após as apresentações, foi realizada uma roda de conversa para cada estudante explanar a sua opinião sobre as cenas, bem como fazer relatos pessoais, caso se sentissem à vontade.

Durante a execução dos módulos, os escolares foram submetidos a três tipos de avaliações. A avaliação somativa correspondeu a dez questões de múltipla escolha sobre os temas contidos no decorrer dos encontros. Essa avaliação foi realizada antes da primeira aula e ao final da última aula de cada módulo. Já a avalição da atividade proposta, de caráter qualitativo, tratava-se da percepção dos alunos quanto aos assuntos, metodologias de ensino-aprendizagem e os recursos utilizados durante as aulas, assim como pela conduta dos tutores e as relações interpessoais durante as aulas. Estas foram realizadas ao final das respectivas aulas, com os seguintes itens: "que bom", "que pena" e "o que precisa melhorar?".

Por fim, foram realizadas também avaliações formativas dos alunos pelos tutores da sala de aula. Essa avaliação teve por objetivo mensurar o desempenho e a performance dos alunos durantes as aulas nos seguintes itens: "capacidade de se relacionar com os membros do grupo"; "capacidade de se relacionar com o orientador/tutor da atividade"; "capacidade de se expressar de forma clara, objetiva e organizada"; "utilização de conhecimentos prévios para embasar seu aprendizado e seus questionamentos"; e "demonstração interesse e habilidades na aprendizagem, desenvolvimento e execução de atividades propostas". Com pontuação máxima de 2 pontos para cada item, totalizando 10 pontos. 
A análise dos dados característicos dos sujeitos foi realizada por meio do software Excel ${ }^{\circledR}$ versão 2004 com abordagem descritiva por intermédio de medidas de tendência central. Os dados não paramétricos das avaliações foram tratados mediante o software BioEstat versão 5.0 com medidas de tendência central e separatrizes, além da análise estatística por meio do Teste de Wilcoxon considerando o IC de $95 \%$ e valor-p $<0,05$.

\section{RESULTADOS}

Entre as 100 vagas disponíveis, apenas 84 discentes assinaram o TCLE e iniciaram as atividades. Entretanto, mediante intercorrências cotidianas e incompatibilidades dos alunos com os horários de aula, apenas 70 discentes participaram do Módulo da Fisiologia do Sistema Cardiorrespiratório. Além disso, para a realização do Módulo da Fisiologia dos Aparelhos Reprodutores Humanos, apenas
20 desses alunos continuaram participando regularmente das atividades e foram incluídos na análise dos dados desse módulo.

Entre as instituições de ensino incluídas, houve maior adesão dos escolares do Colégio Estadual professor Abelardo Romero Dantas com percentil de 54,3\%. Na tabela 1, pode-se visualizar a caracterização dos sujeitos. Em relação à distribuição dos alunos por série, houve maior concentração na terceira série $(50 \%)$ e menor na primeira série $(10 \%)$ do ensino médio. Há que se considerar também a discrepância de participação dos alunos de acordo com o gênero, visto que somente $11 \%$ dos estudantes eram do gênero masculino em comparação a $89 \%$ do gênero feminino.

Tabela 1. Caracterização dos estudantes de ensino médio participantes da cidade de Lagarto/SE - 2018

\begin{tabular}{ll}
\hline Características & FA (\%) \\
\hline Gênero & $62(89 \%)$ \\
Feminino & $8(11 \%)$ \\
Masculino & \\
Séries do Ensino Médio & $7(10 \%)$ \\
1 $^{a}$ série & $28(40 \%)$ \\
$2^{a}$ série & $35(50 \%)$ \\
$3^{a}$ série & \\
\hline
\end{tabular}

Fonte: elaboração própria.

FA: Frequência absoluta. FR: Frequência relativa. 
Conforme a tabela 2, é possível observar aumento significativo das notas na segunda avaliação somativa nos módulos do sistema cardiorrespiratório $(\mathrm{p}<0,0001)$ e dos aparelhos reprodutores $(p=0,0038)$. Destacam-se ainda maiores diferenças entre as notas de avaliação inicial e final, de acordo com os quartis e mediana, no módulo do sistema cardiorrespiratório.

Tabela 2. Resultados das avaliações somativas dos estudantes de ensino médio participantes da cidade de Lagarto/SE - 2018

\begin{tabular}{|c|c|c|c|c|c|c|c|c|}
\hline & \multicolumn{4}{|c|}{$\begin{array}{l}\text { Módulo do Sistema } \\
\text { Cardiorrespiratório }\end{array}$} & \multicolumn{4}{|c|}{ Módulo dos Aparelhos Reprodutores } \\
\hline & $\mathbf{Q}_{1 / 4}$ & Mediana & $Q_{3 / 4}$ & $\begin{array}{l}\text { Z-score } \\
\text { (valor-p) }\end{array}$ & $Q_{1 / 4}$ & Mediana & $\mathbf{Q}_{3 / 4}$ & $\begin{array}{l}\text { Z-score } \\
\text { (valor-p) }\end{array}$ \\
\hline $1^{a}$ avaliação & 1,2 & 2 & 3 & 6,5 & 2 & 3 & 3,2 & 2,9 \\
\hline $2^{a}$ avaliação & 3 & 4 & 5 & $(<0.0001)^{*}$ & 3 & 4 & 4 & $(0.0038)^{*}$ \\
\hline
\end{tabular}

Fonte: elaboração própria.

Q 1/4: primeiro quartil; Q 3/4: terceiro quartil; *: variável apresenta significância estatística $(\mathrm{p}<0,05)$.

Nas avaliações formativas, houve aumentos nas notas finais em ambos os módulos (tabela 3). O módulo do sistema cardiorrespiratório, a partir dos resultados dos quartis e mediana, destacaram-se positivamente $(\mathrm{p}<0,0001)$ em relação às notas do módulo de aparelhos reprodutores $(p=0,0007)$.
Nas análises qualitativas feitas a partir da avaliação da atividade proposta, os alunos relataram pontos positivos das aulas: "a explicação é ótima", "tivemos a oportunidade de aprender", "a aula foi bem elaborada" e "tivemos a oportunidade de aprender na aula". Já em relação ao que não ocorreu da melhor forma nas aulas, os alunos expuseram: "a aula é só uma vez na semana", "a aula foi rápida”, "o horário das aulas precisa ser mais cedo".

Tabela 3. Resultados das avaliações formativas dos estudantes de ensino médio participantes da cidade de Lagarto/SE - 2018

\begin{tabular}{|c|c|c|c|c|c|c|c|}
\hline \multicolumn{4}{|c|}{$\begin{array}{l}\text { Módulo do Sistema } \\
\text { Cardiorrespiratório }\end{array}$} & \multicolumn{4}{|c|}{ Módulo dos Aparelhos Reprodutores } \\
\hline $\mathbf{Q}_{1 / 4}$ & Mediana & $\mathbf{Q}_{3 / 4}$ & $\begin{array}{l}\text { Z-score } \\
\text { (valor-p) }\end{array}$ & $\mathbf{Q}_{1 / 4}$ & Mediana & $\mathbf{Q}_{3 / 4}$ & $\begin{array}{l}\text { Z-score } \\
\text { (valor-p) }\end{array}$ \\
\hline 7 & 7,7 & 8,5 & 5,7 & 7 & 8 & 8.5 & 3,4 \\
\hline 8 & 8,5 & 9,1 & $(<0.0001)^{*}$ & 7,7 & 9 & 9 & $(0.0007)^{\star}$ \\
\hline
\end{tabular}

Fonte: elaboração própria.

Q 1/4: primeiro quartil; Q 3/4: terceiro quartil; *: variável apresenta significância estatística $(\mathrm{p}<0,05)$. 
Quando questionados sobre as sugestões para melhoria das aulas, os alunos responderam: "era bom se tivéssemos outros módulos", "ter mais experiências com massinhas", "as aulas deveriam ser mais dias na semana", "não precisa melhorar nada, está tudo ótimo".

\section{DISCUSSÃO}

Os resultados obtidos por meio deste estudo destacam o efeito positivo da utilização de metodologias inovadoras no ensino da Anatomofisiologia Humana com estudantes do ensino médio. As avaliações somativas denotam aumento considerável das notas em longo prazo traduzido por maior capacidade de assimilação dos conteúdos ao fim dos módulos. Além disso, as avaliações formativas evidenciam melhor desempenho dos estudantes nos domínios de expressões (diálogos, opiniões e questionamentos) e relações interpessoais e com os tutores no decorrer das atividades.

Paulo Freire ${ }^{2}$ propõe o debate a respeito da concepção bancária da educação, contemplada e estruturada mediante narrações e dissertações. Esse modelo nega a pluralidade, o dinamismo da realidade e o contexto reflexivo do que se aprende, de maneira que transpõe o aluno do estado de protagonismo do processo de aprendizado para então tornar-se ouvinte e depositário de narrativas compartimentalizadas pelos educadores $^{2}$.

Dessa forma, há que se considerar a necessidade de ruptura com esse modelo de educar. A instituição de métodos de ensino inovadores pressupõe a mudança dos papéis assumidos por docentes e discentes no processo ensino-aprendizagem, favorecendo a autonomia individual e as habilidades de comunicação dos estudantes6. Nesse sentido, o cenário mundial de pandemia e isolamento social iniciado em 2020 por intermédio da covid-197 tem compulsoriamente impulsionado essa adequação no processo ensino-aprendizagem nas escolas brasileiras, favorecendo o debate e a reflexão sobre os papéis do professor e do aluno na aprendizagem.

Para Cunha et al. ${ }^{8}$, inovar no processo de ensino ultrapassa a introdução de novas tecnologias, logo, esta somente é alcançada a partir da variação nas formas de "ensinar e aprender", conduzidas por meio de um processo emancipatório. Reafirmando essa premissa, as metodologias utilizadas neste estudo propunham a participação ativa dos estudantes no processo de aprendizagem, tornando-os corresponsáveis pela busca do conhecimento à medida que, institivamente, estes tornavam-se autônomos para aquisição do saber. A adoção dessas novas formas de ensino-aprendizagem favorece à democratização do acesso à educação e mudanças no processo de aprendizagem nas condições de isolamento social, visto que elas dispõem ao aluno a independência necessária para sua aprendizagem crítico-reflexiva.

A implementação teórica e prática dos conteúdos de anatomia e fisiologia humana para alunos do ensino médio torna-se um desafio, uma vez que esses conteúdos não são amplamente discutidos em sala de aula. Sendo assim, este estudo assume pioneirismo na abordagem metodológica com esses sujeitos, pois a utilização de métodos de participação ativa, atividades criativas, experimentais e artísticas facilitaram o desempenho dos estudantes durantes as atividades.

No estudo realizado por Rocha9, 72 estudantes de graduação na área saúde foram divididos em dois grupos: um com metodologia ativa de ensino-aprendizagem e outro com metodologia tradicional. No grupo metodologia ativa, foram utilizados recursos como: modelos anatômicos artísticos; jogos didáticos, a exemplo de palavras-cruzadas, quiz, jogos de memória, quebra-cabeça e dominó; além de desenhos artísticos. Já no grupo metodologia tradicional, foram ministradas apenas aulas tradicionais de anatomia humana. Após análises das avaliações antes e após as aulas, foi percebida melhora significativa das notas pós-teste em ambos os grupos.

A introdução de outras formas e instrumentos do aspecto artístico no 
aprendizado é imprescindível para a construção da imagem e da consciência do que se estuda. Assim como neste estudo foram utilizados meios artísticos visuais para o desenvolvimento dos conteúdos, o estudo qualitativo observacional realizado por Silva e Guimarães ${ }^{10}$ utilizou de esculturas, desenhos e pinturas para o aprendizado da anatomia humana e comparada com estudantes de licenciatura do curso de ciências biológicas. Esses autores concluíram haver maior expressão artística dos sujeitos resultante de um desenvolvimento pessoal, assim como melhora no aproveitamento acadêmico pela construção de ambiente harmonioso em sala de aula durante a realização das atividades 9 .

Foureaux e colaboradores ${ }^{1}$ também utilizaram a construção de mapas conceituais para o processo ensino-aprendizagem na disciplina de anatomia humana, durante seis semestres, com o total de 298 graduandos de fisioterapia da Universidade Federal de Minas Gerais. Após a comparação das notas dos sujeitos antes e após a utilização dos mapas, foi percebida redução média das notas finais, entretanto, após sistematização do uso dos mapas na disciplina, foi observado melhora nas pontuações finais, além da redução do índice de reprovação na disciplina ${ }^{1}$.

Por tratar-se de um estudo posto no contexto do desenvolvimento de ensino e aprendizagem, a avaliação é inerente e plural. Segundo Borges e colaboradores ${ }^{11}$, as avaliações somativas objetivam a comprovação da assimilação dos conteúdos pelos alunos, certificando-os a respeito do conteúdo estabelecido. Essa forma de avaliação foi realizada neste trabalho, de maneira que os resultados alcançados na segunda prova atestam eficácia do uso das metodologias inovadoras ao passo que evidenciam inabilidade de alcance de valores máximos nelas. Portanto, Andriola e Araújo ${ }^{12}$ corroboram isso ao afirmar a limitação dessa forma de avaliação no aperfeiçoamento do aprendizado, uma vez que esta é dada após a etapa de formação.

Em contrapartida, a avaliação formativa propõe o aperfeiçoamento do processo ensino-aprendizagem por meio de comentários contínuos sobre o desempenho do aluno ${ }^{13}$. A mensuração do protagonismo e envolvimento dos sujeitos e o trabalho em grupo durante o estudo por intermédio dessa forma de avaliação foi eficaz, já que evidenciou ótimo desempenho dos sujeitos nos domínios previamente estabelecidos, repercutindo em avanço progressivo das notas no decorrer das atividades. Desse modo, Sadler ${ }^{14}$ confirma o emprego da avaliação formativa como instrumento potencializador da transformação positiva entre o que o aluno produz e o que se espera no processo de aprendizagem.

A avaliação da atividade proposta direcionada ao feedback dos sujeitos sobre as metodologias de ensino-aprendizagem foi ímpar ao favorecer o aprimoramento das metodologias utilizadas a cada encontro. Dessa maneira, garantiu variabilidade das atividades e dinâmicas desenvolvidas, assim como na autopercepção dos sujeitos a respeito do desenvolvimento de cada aula, logo, concebendo o processo de aprendizagem em que está inserido.

Este estudo extensionista fez parte do processo de divulgação e fortalecimento da utilização de metodologias ativas no ensino superior no interior do estado de Sergipe por meio da UFS. Além disso, favoreceu a aproximação das realidades do ensino médio e superior, como também a construção de uma visão crítico-reflexiva a partir do uso dessas metodologias para, principalmente, instigá-los ao ingresso no ensino superior.

Ademais, mediante o presente estudo, foi possível ressaltar a necessidade e a importância de adaptações nos métodos avaliativos em sala de aula, a fim de garantir a integração dos estudantes com o grupo e os docentes, além de favorecer o aperfeiçoamento constante do processo ensino-aprendizagem; contribuindo assim, para o rompimento com o modelo bancário de ensino imposto tradicionalmente.

Por fim, a limitação deste estudo se deu pela ausência de um grupo controle com o uso de métodos de ensino tradicionais. 


\section{CONCLUSÕES}

Pelo presente estudo, pôde-se constatar a eficácia da utilização das metodologias ativas e seus diversos recursos para a construção de uma visão crítico-reflexiva dos estudantes, assim como para o ensino da anatomia humana, por meio da melhora no desempenho dos alunos nas avaliações somativas e formativas durante a execução dos módulos.

Sendo assim, é fundamental que se incorpore na educação o uso de metodologias inovadoras no processo ensino-aprendizagem, considerando, pincipalmente, a realidade de ensino remoto no cenário educacional e democratização do acesso à educação no período de isolamento social, de modo a contribuir para a construção do conhecimento partilhado e exercício da autonomia na construção do conhecimento dos estudantes.

\section{REFERÊNCIAS}

1. Foureaux G, Sá MA, Schetino LP, Guerra LB, Silva JH. O ensino-aprendizagem da anatomia humana: avaliação do desempenho dos alunos após a utilização de mapas conceituais como estratégia pedagógica. Ciênc Educ. 2018;24(1)95-110.

2. Freire P. Pedagogia da autonomia. 17. ed. Rio de Janeiro: Paz e Terra; 1987.

3. Behrens MA. A prática pedagógica e o desafio do paradigma emergente. R Bras Est Pedag.1999;80(196):383-403.

4. Mitre SM, Siqueira-Batista R, Girardi-de-Mendonça JM, Morais-Pinto NM, Meirelles CAB, Pinto-Porto C, et al. Metodologias ativas de ensino-aprendizagem na formação profissional em saúde: debates atuais. Ciênc Saúde Coletiva. 2008;13 Sup(2):2133-2144.
5. Aguiar RG, Barbosa GR, Oliveira AMB, Mocellin AS, Costa MRCD, Silveira NA. Implantação de um curso de fisioterapia baseado em metodologias ativas de ensino-aprendizagem. Cad Edu Saúde e Fis. 2014;1(1):13-18.

6. Souza CS, Iglesias AG, Pazin-Filho A. Estratégias inovadoras para métodos de ensino tradicionais - aspectos gerais. Medicina (Ribeirão Preto). 2014;24(3):284-92.

7. Pascarella G, Strumia A, Piliego C, Bruno F, Del Buono R, Costa F, et al. COVID-19 diagnosis and management: a comprehensive review. J Intern Med. 2020;288(2):192-206.

8. Cunha MI, Marsico HL, Borges FA, Tavares P. Inovações pedagógicas na formação inicial de professores. In: Fernandes CMB, Grillo M, organizadores. Educação superior: travessias e atravessamentos. Canoas: Editora da ULBRA; 2001.

9. Rocha DP. Alternativas didático-pedagógicas para auxílio no estudo da anatomia humana no ensino superior [dissertação]. Petrolina (PE): Universidade de Pernambuco campus Petrolina; 2017.

10. Silva RA, Guimarães MM. Arte educação: facilitando o ensino de morfologia. Educere. 2004;4(1):55-63.

11. Borges MC, Miranda CH, Santana RC, Bollela VR. Avaliação formativa e feedback como ferramenta de aprendizado na formação de profissionais da saúde. Medicina (Ribeirão Preto). 2014;47(3):324-31.

12. Andriola WB, Araújo AC. Potencialidades da avaliação formativa e somativa. Rev Eletrônica Acta Sapientia. 2018;5(1):1-15.

13. Souza RGS. Atributos fundamentais dos procedimentos de avaliação. In: Tibério IFLC, Daud-Gallotti RM, Troncon LEA, Martins MA. Avaliação prática de habilidades clínicas em medicina. São Paulo: Atheneu; 2012. p. 1-12.

14. Sadler DR. Formative assessment and the design of instructional systems. Instructional Science. 1989;18(1)145-65. 